\title{
Comparison of MANET Routing Protocol-A Review
}

\author{
Ramandeep, Sangeeta Monga \\ M.Tech Scholar (ECE Department) DAV University Jalandhar \\ Assistance Prof. (ECE Department) DAV University Jalandhar
}

\begin{abstract}
Mobile adhoc network is a collection of wireless mobile hosts forming a temporary network without the aid of any standalone infrastructure or centralized administration.MANET routing algorithm is necessary to find specific routes between source and destination.The primary goal of any adhoc network routing protocol is to meet the challenges of the dynamically changing topology and establish an efficient route between any two nodes with minimum delay androuting overheads. Therefore, it is difficult to determine which protocol performs best under a number of different scenarios. The aim of this paper is to compare various routing protocols qualitatively.
\end{abstract}

Keywords: $A O D V, D S R, D S D V, C G S R, W R P, M A N E T$.

\section{Introduction:}

Adhoc wireless network is a collection of two or more devices with wireless communication and networking capability. A wireless device can be different forms (for example: palmtop, laptop, internet mobile phoneetc). The MANETs are autonomously self organized and self configuring networks without infrastructure support. In such networks since node mobility is very high the network may experience frequent topology changes, making routing a challenging task in mobile Adhoc networks. Mobility and absence of any fixed infrastructure make MANETs very attractive for various applications like students using laptop to participate in an interactive lecture, business associates sharing informationduring a conference, search and rescue operation, emergency services, entertainment, sensornetworks and commercial environments.

MANETs can be developed quickly at a very lowcost and can be easily managed. In the future there is no doubt that we will have more and more adhoc networks in which routing is one of the critical issue. Need of routing algorithm arises whenever a packet needs to be transmitted to node via number of different nodes. Section2 describes the classification of MANET protocols section3 compares the various protocol qualitatively and section4gives the conclusion and future scope. [1,2]

\section{Classification Of Routing Protocol:}

2.1 Table driven routing protocol: It is also known as proactive routing protocol. In this protocol each and every node maintain complete information about network topology by continuously evaluating route to all the nodesand these table driven routing protocol attempts to maintain consistent, up to date routing information from each node to every node in the network.

2.2 On-demand routing protocol: It is also known as source initiated and reactive routing protocol. This type of routing creates routes only when desired by the source node. When a node requires a route to a destination, it initiates a route discovery process within the network. This process is completed once a route is found or all possible routepermutations have been examined. Once a route has been discovered and established, it is maintained by some form of route maintenance procedure until either the destination becomes inaccessible along every path from the source or the route is no longer desired.

2.3 Hybrid routing protocol: Hybrid routing protocol use both methods of proactive and reactive protocol .In these protocols proactive operations are restricted to small domain whereas reactive protocols are used for locating nodes outside those domains. [1,2,7] Comparison of these is given in table 1

TABLE 1: Comparison of Table driven, On demand and Hybrid Protocols $[3,4,9]$

\begin{tabular}{|l|l|l|l|l|}
\hline S.no & Parameters & $\begin{array}{l}\text { Table driven (Proactive) routing } \\
\text { protocol }\end{array}$ & $\begin{array}{l}\text { On-demand (Reactive) routing } \\
\text { protocol }\end{array}$ & Hybrid routing protocol \\
\hline 1 & Network structure & Flat and hierarchical & Flat & Flat and hierarchical \\
\hline 2 & $\begin{array}{l}\text { Topology } \\
\text { dissemination }\end{array}$ & Periodical & On-demand & Both \\
\hline 3 & Storage requirements & High & Usually lower than proactive & Medium \\
\hline 4 & Route formation delay & Minimum & Maximum & Medium \\
\hline 5 & Route availability & Always available & Available when needed & $\begin{array}{l}\text { Routes are available in intra } \\
\text { zone }\end{array}$ \\
\hline 6 & Communication overhead & High & Low & Medium \\
\hline 7 & Types of protocols & DSDV,CGSR,WRP,GRP,GSRetc. & $\begin{array}{l}\text { AODV,DSR,TORA, } \\
\text { ABR, SSRetc. }\end{array}$ & ZRPR \\
\hline
\end{tabular}




\section{Comparison of various protocols:}

Comparison of various protocols such as DSDV, CSGR, WRP, AODV and DSR is given in table $2:[1,2,4,5,6,7,8,9]$

TABLE 2: Comparison of DSDV, CSGR, WRP, AODV and DSR Protocols

\begin{tabular}{|c|c|c|c|c|c|c|}
\hline S.No & Parameters & DSDV & CSGR & WRP & AODV & DSR \\
\hline 1 & Protocol type & $\begin{array}{l}\text { Destination sequence } \\
\text { distance vector }\end{array}$ & $\begin{array}{l}\text { Cluster switch } \\
\text { gateway routing }\end{array}$ & $\begin{array}{l}\text { Wireless routing } \\
\text { protocol }\end{array}$ & $\begin{array}{lr}\begin{array}{l}\text { Adhoc on } \\
\text { distance } \\
\text { routing }\end{array} & \text { vemand } \\
\text { vector }\end{array}$ & $\begin{array}{l}\text { Dynamic } \\
\text { source routing }\end{array}$ \\
\hline 2 & $\begin{array}{l}\text { Routing } \\
\text { approaches }\end{array}$ & Proactive & Proactive & Proactive & Reactive & Reactive \\
\hline 3 & $\begin{array}{l}\text { Routing } \\
\text { structure }\end{array}$ & Flat structure & $\begin{array}{l}\text { Hierarchical } \\
\text { Structure }\end{array}$ & Flat structure & Flat structure & Flat structure \\
\hline 4 & $\begin{array}{l}\text { Route } \\
\text { selection }\end{array}$ & Link state & Shortest path & Shortest path & $\begin{array}{l}\text { Shortest and updated } \\
\text { path }\end{array}$ & $\begin{array}{l}\text { Shortest and } \\
\text { updated path }\end{array}$ \\
\hline 5 & Route & Single route & $\begin{array}{l}\text { Single and multiple } \\
\text { route }\end{array}$ & Single route & Multiple route & Multiple route \\
\hline 6 & Routing table & $\begin{array}{l}\text { Each node maintain } \\
\text { a complete address } \\
\text { to each destination }\end{array}$ & $\begin{array}{l}\text { Two table } \\
\text { 1.Routing table } \\
\text { 2.Cluster member } \\
\text { table }\end{array}$ & $\begin{array}{l}\text { Four tables } \\
\text { 1.Distance table } \\
\text { 2.Routing table } \\
\text { 3.Link cost table } \\
\text { 4.message } \\
\text { retransmission list }\end{array}$ & $\begin{array}{l}\text { Each node maintain a } \\
\text { route table in which } \\
\text { next hop routing } \\
\text { information for } \\
\text { destination node is } \\
\text { stored }\end{array}$ & $\begin{array}{l}\text { Route cache } \\
\text { Full route to } \\
\text { destination }\end{array}$ \\
\hline 7 & $\begin{array}{l}\text { Route } \\
\text { maintenance }\end{array}$ & $\begin{array}{l}\text { Each node in the } \\
\text { mobile network } \\
\text { maintains a routing } \\
\text { table in which all the } \\
\text { routes to possible } \\
\text { destination with non- } \\
\text { partitioned network } \\
\text { and number of hops } \\
\text { to each destination } \\
\text { are recorded }\end{array}$ & $\begin{array}{l}\text { Each node maintains } \\
\text { a routing table which } \\
\text { is used to determine } \\
\text { the next hop to reach } \\
\text { the destination. }\end{array}$ & $\begin{array}{l}\text { Routing node } \\
\text { maintains the distance } \\
\text { and second to last hop } \\
\text { information for each } \\
\text { destination in the } \\
\text { wireless network. }\end{array}$ & $\begin{array}{l}\text { Every node maintains } \\
\text { two counters } \\
\text { Sequence no and } \\
\text { broadcast ID. }\end{array}$ & $\begin{array}{l}\text { Two different } \\
\text { processes: } \\
\text { 1.Hop by hop } \\
\text { acknowledge } \\
\text { ment at the } \\
\text { data link layer } \\
\text { 2.End to end } \\
\text { acknowledge } \\
\text { ment }\end{array}$ \\
\hline 8 & $\begin{array}{l}\text { Source } \\
\text { routing }\end{array}$ & $\begin{array}{l}\text { It has no source } \\
\text { routing. }\end{array}$ & No may be possible. & $\begin{array}{l}\text { It has no source } \\
\text { routing. }\end{array}$ & $\begin{array}{l}\text { It has no source } \\
\text { routing. }\end{array}$ & $\begin{array}{l}\text { It has possible } \\
\text { source } \\
\text { routing. }\end{array}$ \\
\hline 9 & $\begin{array}{l}\text { Hello } \\
\text { message } \\
\text { required }\end{array}$ & Yes & Yes & Yes & Yes & No \\
\hline 10 & $\begin{array}{l}\text { Operation of } \\
\text { protocols }\end{array}$ & $\begin{array}{l}\text { Routing information } \\
\text { is always available, } \\
\text { whether the source } \\
\text { node require a route } \\
\text { or not because each } \\
\text { node in the mobile } \\
\text { network maintains a } \\
\text { routing table. }\end{array}$ & $\begin{array}{l}\text { Mobile nodes are } \\
\text { grouped into cluster } \\
\text { and each cluster has } \\
\text { cluster head and } \\
\text { cluster head to } \\
\text { gateway routing } \\
\text { approach to move } \\
\text { traffic from source to } \\
\text { destination. Gateway } \\
\text { nodes are } \\
\text { communication } \\
\text { range of two or more } \\
\text { cluster head. }\end{array}$ & $\begin{array}{l}\text { In WRP, routing } \\
\text { nodes communicate } \\
\text { the distance and } \\
\text { second to last hop } \\
\text { information for each } \\
\text { destination in wireless } \\
\text { network and it belong } \\
\text { to path finding } \\
\text { algorithm. }\end{array}$ & $\begin{array}{l}\text { 1.RREQ broadcast } \\
\text { 2.RREP Propagation } \\
\text { 3.RERR message }\end{array}$ & $\begin{array}{l}\text { 1.RREQ } \\
\text { broadcast } \\
\text { 2.RREP } \\
\text { Propagation } \\
\text { 3.RERR } \\
\text { message }\end{array}$ \\
\hline 11 & Advantages & $\begin{array}{l}\text { 1.Loop free } \\
\text { 2. Shortest path to } \\
\text { every destination is } \\
\text { chosen. }\end{array}$ & $\begin{array}{l}\text { 1.Cluster head can } \\
\text { control a group of } \\
\text { adhoc hosts. } \\
\text { 2. Cluster provide a } \\
\text { framework for code } \\
\text { separations, channel } \\
\text { access, routing, } \\
\text { bandwidth } \\
\text { allocation. }\end{array}$ & $\begin{array}{l}\text { 1. Avoid the count to } \\
\text { infinity problems by } \\
\text { forcing each node to } \\
\text { perform consistency } \\
\text { checks. } \\
2 \text {. Routing } \\
\text { information is } \\
\text { accurate, mobile send } \\
\text { updates messages } \\
\text { periodically to their } \\
\text { neighbors. }\end{array}$ & $\begin{array}{l}\text { 1.Adaptable to high } \\
\text { dynamic topology. } \\
\text { 2. loop free } \\
3 \text { AODV has higher } \\
\text { bandwidth efficiency } \\
\text { because of lesser } \\
\text { overheads }\end{array}$ & $\begin{array}{l}\text { 1.Support } \\
\text { Multipath } \\
\text { routing }\end{array}$ \\
\hline 12 & Limitation & $\begin{array}{l}\text { 1.High overhead 2.It } \\
\text { doesn't support } \\
\text { multipath routing }\end{array}$ & $\begin{array}{l}\text { 1. If a cluster head is } \\
\text { changing frequently } \\
\text { and nodes will be } \\
\text { spending a lot of } \\
\text { time converging to a } \\
\text { cluster head. }\end{array}$ & $\begin{array}{l}\text { 1. More overheads are } \\
\text { required due to 'hello' } \\
\text { messages. }\end{array}$ & $\begin{array}{l}\text { 1. Scalability problems } \\
\text { due to large delay } \\
\text { 2.AODV takes more } \\
\text { time to build the } \\
\text { routing table. }\end{array}$ & $\begin{array}{l}1 \text { Scalability } \\
\text { problems due } \\
\text { to source } \\
\text { routing and } \\
\text { flooding. } \\
2 . \quad \text { Being a } \\
\text { reactive } \\
\text { protocol DSR } \\
\text { suffers from } \\
\text { high route } \\
\text { discovery } \\
\text { latency. }\end{array}$ \\
\hline
\end{tabular}




\section{Conclusion and Future Scope:}

Mobile adhoc network is decentralized, selforganized, anytime, anywhere networks and provide cheap communication. In this paper classification of routing protocols on the basis of routing information updates mechanism, is discussed and comparative analysis of mobile Adhoc networks routing protocol like: DSDV, CGSR, WRP, AODV and DSR is done. Each protocol has its own advantages and disadvantages and it's well suited for particular situations. However because of their advantages, wireless Adhoc networks are becoming more and more prevalent in the world. So lot of research can be done in routing protocols of MANET.

\section{Reference:}

[1]. Ck Toh, “Adhoc Mobile Wireless Networks: Protocol and System”, Pearson, India, 2008

[2]. Stefano Basagni, Marco Conti ,Silvia Giordano, Ivan Stojmenovic, "Mobile Ad Hoc Networking”, IEEE Press, Wiley India , 2010

[3]. Marco Conti, “Multihop Adhoc Networking The Theory," SUPSI,April 2007

[4]. Charu Wahi, Sanjay Kumar Sonbhadra, "Mobile Adhoc Network Routing Protocols",IJASUC, Vol3,No.2, April 2012

[5]. file:///F:/Comparison\%20between\%20aodv\%20and\%20olsr\%20protocol.htm

[6]. http://en.wikipedia.org/wiki/Mobile_adhoc_network

[7]. K.Prabu, A.Subramani, "Performance Comparison of Routing Protocols in MANET,'IJARCSSE,Vol.2, Issue9,September 2012

[8]. Anuj k.Gupta, Harsh Sadawarti, Anil K.Verma, "Performance Analysis of AODV, DSR, TORA Routing Protocol", IACSIT, Vol.2,No.2,April 2010

[9]. Rakesh kumar, Piush Verma, Yaduvir Singh, "Comparision of MANET Protocols With Secure Routing Scheme Developed Using Evolution Algorithms”,IJCET,Volume 3,Issue 2,September 2012 